\begin{tabular}{|c|c|}
\hline Title & V ectorial wave analysis of dielectric waveguides for optical-integrated circuits using equivalent network approach \\
\hline Author(s) & Koshiba, M.; Suzuki, M. \\
\hline Citation & Journal of Lightwave Technology, 4(6), 656-664 \\
\hline Issue Date & 1986-06 \\
\hline Doc URL & http:/hdl.handle.net/2115/7373 \\
\hline Rights & $\begin{array}{l}\text { (C1986 IEEE. Personal use of this material is permitted. However, permission to reprint/republish this material for } \\
\text { advertising or promotional purposes or for creating new collective works for resale or redistribution to servers or lists, } \\
\text { or to reuse any copyrighted component of this work in other works must be obtained from the IEEE. } \\
\text { IEEE, Journal of Lightwave Technology, } 4(6), 1986, \text { p656-664 }\end{array}$ \\
\hline Type & article \\
\hline File Information & JLT4_6.pdf \\
\hline
\end{tabular}

Instructions for use 


\title{
Vectorial Wave Analysis of Dielectric Waveguides for Optical-Integrated Circuits Using Equivalent Network Approach
}

\author{
MASANORI KOSHIBA, SENIOR MEMBER, IEEE, AND MICHIO SUZUKI, SENIOR MEMBER, IEEE
}

\begin{abstract}
A vectorial wave analysis of the propagation characteristics of open dielectric waveguides for optical-integrated circuits using an equivalent network approach is presented. In this approach, all of the contributions from the discrete and continuous parts of the spectrum and from the TE-TM coupling, which are neglected in the earlier equivalent network approach, are taken into account. To show the validity and usefulness of this formulation, examples are computed for optical strip waveguides, rib waveguides, rectangular dielectric waveguides, embossed waveguides, and embedded waveguides.
\end{abstract}

\section{INTRODUCTION}

$\mathrm{O}$ PEN DIELECTRIC WAVEGUIDES for optical-integrated circuits as shown in Fig. 1 , where $n_{j}^{(i)}(i=$ $1,2$ and $j=1,2,3,4)$ is the refractive index and $n_{3}^{(i)} \geq n_{4}^{(i)}$, are very difficult to analyze rigorously. Some analyses of optical strip waveguides, rib waveguides, rectangular dielectric waveguides, embossed waveguides, and embedded waveguides obtained from the somewhat generalized waveguide structure in Fig. 1 have been attempted through the following approaches [1]: the approximate analytical approach [2]-[4]; and the numerical approach [5]-[8]. Although the calculation procedure of the former approach is relatively simple, its accuracy is practically insufficient. On the other hand, the latter approach is more effective for precise investigation. However, the numerical approach is, in general, time consuming and expensive. Recently, the equivalent network approach has been developed for open dielectric waveguides [9]-[16]. The calculation procedure of this approach is relatively simple, and application of the socalled transverse resonance technique yields approximate but fairly accurate analytical expressions for the dispersion relations for the propagation wavenumber $k_{z}$ in the $z$ direction. However, since the continuous spectrum contributions at the sides of the waveguide are neglected, the equivalent network representation cannot be derived for the waveguides having no discrete modes in region 2 in Fig. 1, such as the rectangular dielectric waveguide, the embossed waveguide, and the embedded waveguide [14]. For the analysis of a dielectric waveguide on a ground plane, Koshiba et al. [17], [18] have presented an im-

Manuscript received September 3, 1985; revised January 16, 1986. The authors are with the Department of Electronic Engineering, Hokkaido University, Sapporo, 060 Japan.

IEEE Log Number 8608007.

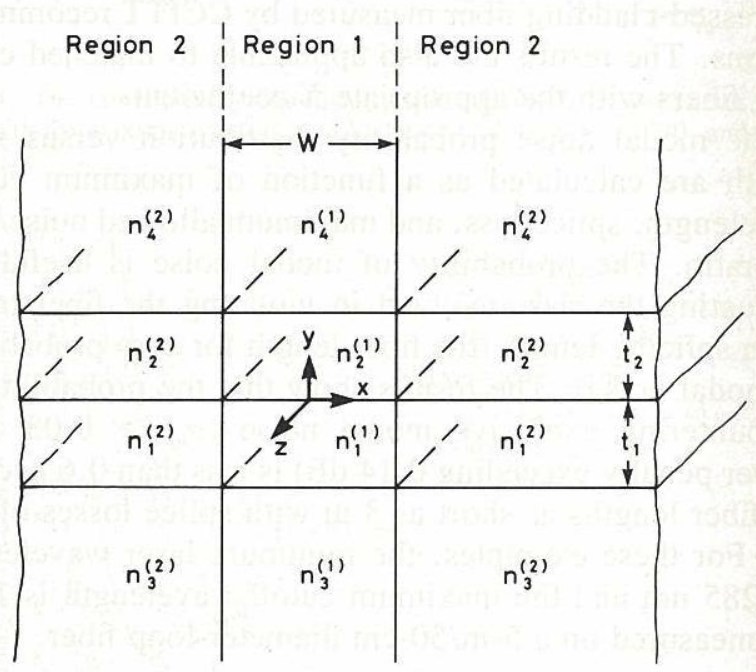

Fig 1. Dielectric waveguide for optical-integrated circuits.

proved equivalent network approach in which the continuous spectrum contributions are taken into account. However, this approach is based on the scalar wave approximation, in which the coupling produced between TE $\left(E_{y} \equiv 0\right)$ and TM $\left(H_{y} \equiv 0\right)$ modes [8], [12], [13] at the sides of the waveguide is neglected. In [12] and [13], the TE-TM coupling is taken into account, but the approximate bounded approach, in which the open dielectric waveguide is enclosed by perfect electric conductors, is used.

This paper presents a more rigorous method of the vectorial wave analysis of open dielectric waveguides for optical-integrated circuits using an equivalent network approach. In this analysis, all of the contributions from the discrete and continuous parts of the spectrum and from the TE-TM coupling are taken into account. To show the validity and usefulness of this formulation, examples are computed for optical strip waveguides, rib waveguides, rectangular dielectric waveguides, embossed waveguides, and embedded waveguides.

\section{Transmission Line Equations}

For the purpose of the analysis, the waveguide is divided into two regions (regions 1 and 2) as shown in Fig. 1 , and then, with respect to the $x$ direction, we express the transverse fields [12], [13] in terms of all TE $\left(E_{y} \equiv\right.$ 
$0)$ and TM $\left(H_{y} \equiv 0\right)$ modes [8], [12], [13], as follows:

$$
\begin{aligned}
E_{t}^{(i)}= & {\left[E_{y}^{(i)} E_{z}^{(i)}\right]^{T} } \\
= & \exp \left(-j k_{z} z\right) \sum_{r=1}^{2}\left\{\sum_{q} V_{r q}^{(i)}(x) f_{r q}^{(i)}(y)\right. \\
& \left.+\sum_{s=0}^{2} \int_{\xi_{s}^{(i)}}^{\eta_{s}^{(i)}} V_{r s}^{(i)}(x, \rho) f_{r s}^{(i)}(y, \rho) d \rho\right\} \\
H_{t}^{(i)}= & {\left[H_{y}^{(i)} H_{z}^{(i)}\right]^{T} } \\
= & \exp \left(-j k_{z} z\right) \sum_{r=1}^{2}\left\{\sum_{q} I_{r q}^{(i)}(x) g_{r q}^{(i)}(y)\right. \\
& \left.+\sum_{s=0}^{2} \int_{\xi_{s}^{(i)}}^{\eta_{s}^{(i)}} I_{r s}^{(i)}(x, \rho) g_{r s}^{(i)}(y, \rho) d \rho\right\}
\end{aligned}
$$

where the superscripts $i=1$ and 2 denote quantities for regions 1 and 2 , respectively, the subscripts $r=1$ and 2 denote quantities for the TE and TM modes, respectively, $T$ denotes a transpose, the summation $\Sigma_{q}$ extends over the discrete modes, $\rho$ is the wavenumber in the $y$-direction of the continuous spectrum inside the medium with the refractive index $n_{3}^{(i)}$, and $\xi_{s}^{(i)}$ and $\eta_{s}^{(i)}$ are given by

$$
\begin{aligned}
& \xi_{s}^{(i)}= \begin{cases}0, & s=0 \\
k_{0} \sqrt{\left(n_{3}^{(i)}\right)^{2}-\left(n_{4}^{(i)}\right)^{2},} & s=1,2\end{cases} \\
& \eta_{s}^{(i)}= \begin{cases}k_{0} \sqrt{\left(n_{3}^{(i)}\right)^{2}-\left(n_{4}^{(i)}\right)^{2},} & s=0 \\
\infty, & s=1,2 .\end{cases}
\end{aligned}
$$

Here $k_{0}$ is the wavenumber of a vacuum. The radiation mode indicated by $s=0$ decays exponentially in the region of refractive index $n_{4}^{(i)}\left(t_{2} \leq y\right)$, since $n_{3}^{(i)} \geq n_{4}^{(i)}$ [19]. For

$$
k_{0} \sqrt{\left(n_{3}^{(i)}\right)^{2}-\left(n_{4}^{(i)}\right)^{2}} \leq \rho
$$

there are two independent types of radiation modes [19]. These radiation modes indicated by $s=1$ and $s=2$ consist of standing waves above and below the core region $\left(-t_{1} \leq y \leq t_{2}\right)[19]$.

The mode functions $f_{r q}^{(i)}(y)$ and $g_{r q}^{(i)}(y)$ for discrete modes and the mode functions $f_{r s}^{(i)}(y, \rho)$ and $g_{r s}^{(i)}(y, \rho)$ for continuous spectrum can be normalized in accordance with the orthonormality statement

$$
\begin{aligned}
\int_{-\infty}^{\infty} & \left\{i_{x} \times f_{r q}^{(i)}(y)\right\}^{*} \cdot g_{r^{\prime} q^{\prime}}^{(i)}(y) d y \\
= & \int_{-\infty}^{\infty}\left\{g_{r q}^{(i)}(y) \times i_{x}\right\}^{*} \cdot f_{r^{\prime} q^{\prime}}^{(i)}(y) d y=\delta_{r r^{\prime}} \delta_{q q^{\prime}}
\end{aligned}
$$

$$
\begin{aligned}
\int_{-\infty}^{\infty} & \left\{i_{x} \times f_{r s}^{(i)}(y, \rho)\right\}^{*} \cdot g_{r^{\prime} s}^{(i)}\left(y, \rho^{\prime}\right) d y \\
= & \int_{-\infty}^{\infty}\left\{g_{r s}^{(i)}(y, \rho) \times i_{x}\right\}^{*} \cdot f_{r^{\prime} s}^{(i)}\left(y, \rho^{\prime}\right) d y \\
= & \delta_{r r^{\prime}} \delta\left(\rho-\rho^{\prime}\right)
\end{aligned}
$$

where the asterisk denotes complex conjugate, $i_{x}$ is the unit vector in the $x$-direction, and $\delta_{q q^{\prime}}$ and $\delta\left(\rho-\rho^{\prime}\right)$ are the Kronecker $\delta$ and the Dirac $\delta$ function, respectively.

The modal voltage $V_{r q}^{(i)}(x)$ and current $I_{r q}^{(i)}(x)$ for discrete modes satisfy the following transmission line equations:

$$
\begin{aligned}
-d V_{r q}^{(i)}(x) / d x & =j \kappa_{r q}^{(i)} Z_{r q}^{(i)} I_{r q}^{(i)}(x) \\
-d I_{r q}^{(i)}(x) / d x & =j \kappa_{r q}^{(i)} Y_{r q}^{(i)} V_{r q}^{(i)}(x) \\
\kappa_{r q}^{(i)} & =\sqrt{\left(k_{r q}^{(i)}\right)^{2}-k_{z}^{2}} \\
Z_{r q}^{(i)} & =1 / Y_{r q}^{(i)}= \begin{cases}\kappa_{1 q}^{(i)} / \omega \epsilon_{1 q}^{(i)}, & r=1 \\
\omega \mu_{2 q}^{(i)} / \kappa_{2 q}^{(i)}, & r=2\end{cases} \\
\epsilon_{1 q}^{(i)} & =\epsilon_{0}\left(k_{1 q}^{(i)} / k_{0}\right)^{2} \\
\mu_{2 q}^{(i)} & =\mu_{0}\left(k_{2 q}^{(i)} / k_{0}\right)^{2}
\end{aligned}
$$

where $\omega$ is the angular frequency, $\epsilon_{0}$ and $\mu_{0}$ are the permittivity and the permeability of a vacuum, respectively, and $k_{r q}^{(i)}$ is the wavenumber of discrete modes for the equivalent layered structure that is uniform along $x$ and $z$.

On the other hand, the modal voltage $V_{r s}^{(i)}(x, \rho)$ and current $I_{r s}^{(i)}(x, \rho)$ for continuous spectrum satisfy the following transmission line equations:

$$
\begin{aligned}
& -d V_{r s}^{(i)}(x, \rho) / d x=j \kappa_{r s}^{(i)}(\rho) Z_{r s}^{(i)}(\rho) \dot{I}_{r s}^{(i)}(x, \rho) \\
& -d I_{r s}^{(i)}(x, \rho) / d x=j \kappa_{r s}^{(i)}(\rho) Y_{r s}^{(i)}(\rho) V_{r s}^{(i)}(x, \rho) \\
& \kappa_{r s}^{(i)}(\rho)=\sqrt{k_{0}^{2}\left(n_{3}^{(i)}\right)^{2}-k_{z}^{2}-\rho^{2}} \\
& Z_{r s}^{(i)}(\rho)=1 / Y_{r s}^{(i)}(\rho)= \begin{cases}\kappa_{1 s}^{(i)}(\rho) / \omega \epsilon_{1 s}^{(i)}(\rho), & r=1 \\
\omega \mu_{2 s}^{(i)}(\rho) / \kappa_{2 s}^{(i)}(\rho), & r=2\end{cases} \\
& \begin{aligned}
\epsilon_{1 s}^{(i)}(\rho) & =\left\{k_{0}^{2}\left(n_{3}^{(i)}\right)^{2}-\rho^{2}\right\} / \omega^{2} \mu_{0} \\
\mu_{2 s}^{(i)}(\rho) & =\left\{k_{0}^{2}\left(n_{3}^{(i)}\right)^{2}-\rho^{2}\right\} / \omega^{2} \epsilon_{0} .
\end{aligned}
\end{aligned}
$$

A summary of the mode functions for discrete modes and continuous spectrum is given in the Appendix.

\section{Equivalent Network for Step Junction}

The dielectric waveguide as shown in Fig. 1 will support the propagation of waves having two possible field configurations, classified as the $E_{p q}^{x}$ and $E_{p q}^{y}$ modes [2] that can be represented by a linear combination of the TE $\left(E_{y} \equiv 0\right)$ and $\mathrm{TM}\left(H_{y} \equiv 0\right)$ modes [8]. The main field components of the members of the first family are $E_{x}$ and $H_{y}$, while those of the second are $E_{y}$ and $H_{x}$. The subscripts $p$ and $q$ indicate the number of extrema of the electric or magnetic field in the $x$ and $y$ directions, respectively.

\section{A. $E_{p q}^{x}$ Modes}

The boundary conditions at each junction plane $(x=$ $\pm W / 2$ ) are that both $E_{t}$ and $H_{t}$ are continuous across it. 


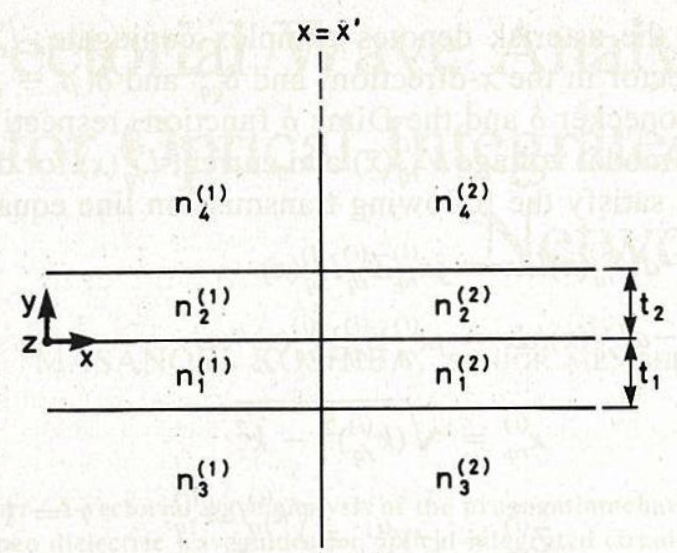

Fig. 2. Step junction between two regions.

Let us assume the unknown magnetic field in the junction plane $x=x^{\prime}$ as shown in Fig. 2 to be $h_{t}$. From (2), the boundary condition for $H_{t}$ gives

$$
\begin{aligned}
h_{t}= & \sum_{r=1}^{2}\left\{\sum_{q} I_{r q}^{(1)}\left(x^{\prime}\right) g_{r q}^{(1)}(y)\right. \\
& \left.+\sum_{s=0}^{2} \int_{\xi_{s}^{(1)}}^{\eta_{s}^{(1)}} I_{r s}^{(1)}\left(x^{\prime} ; \rho\right) g_{r s}^{(1)}(y, \rho) d \rho\right\} \\
= & \sum_{r=1}^{2}\left\{\sum_{q} I_{r q}^{(2)}\left(x^{\prime}\right) g_{r q}^{(2)}(y)\right. \\
& \left.+\sum_{s=0}^{2} \int_{\xi_{s}^{(2)}}^{\eta_{s}^{(2)}} I_{r s}^{(2)}\left(x^{\prime}, \rho\right) g_{r s}^{(2)}(y, \rho) d \rho\right\}
\end{aligned}
$$

Applying (4) to (9), we obtain

$$
\begin{aligned}
I_{r q}^{(i)}\left(x^{\prime}\right) & =\int_{-\infty}^{\infty}\left\{i_{x} \times f_{r q}^{(i)}(y)\right\}^{*} \cdot h_{t} d y \\
I_{r s}^{(i)}\left(x^{\prime}, \rho\right) & =\int_{-\infty}^{\infty}\left\{i_{x} \times f_{r s}^{(i)}(y, \rho)\right\} * \cdot h_{t} d y .
\end{aligned}
$$

From (1), the boundary condition $E_{t}$ gives

$$
\begin{aligned}
\sum_{s=1}^{2}\left\{\sum_{q}\right. & V_{r q}^{(1)}\left(x^{\prime}\right) f_{r q}^{(1)}(y) \\
& \left.+\sum_{s=0}^{2} \int_{\xi_{s}^{(1)}}^{\eta_{s}^{(1)}} V_{r s}^{(1)}\left(x^{\prime}, \rho\right) f_{r s}^{(1)}(y, \rho) d \rho\right\} \\
= & \sum_{r=1}^{2}\left\{\sum_{q} V_{r q}^{(2)}\left(x^{\prime}\right) f_{r q}^{(2)}(y)\right. \\
& \left.+\sum_{s=0}^{2} \int_{\xi_{s}^{(2)}}^{\eta_{s}^{(2)}} V_{r s}^{(2)}\left(x^{\prime}, \rho\right) f_{r s}^{(2)}(y, \rho) d \rho\right\}
\end{aligned}
$$

cross section, we obtain

$$
\begin{aligned}
\sum_{r=1}^{2}\left(\sum_{q} V_{r q}^{(1)}\left(x^{\prime}\right) \int_{-\infty}^{\infty} h_{t}^{*} \cdot\left\{i_{x} \times f_{r q}^{(1)}(y)\right\} d y\right. \\
+\sum_{s=0}^{2} \int_{\xi_{s}^{(1)}}^{\eta_{s}^{(1)}}\left[V_{r s}^{(1)}\left(x^{\prime}, \rho\right) \int_{-\infty}^{\infty} h_{t}^{*}\right. \\
\left.\left.\cdot\left\{i_{x} \times f_{r s}^{(1)}(y, \rho)\right\} d y\right] d \rho\right) \\
=\sum_{r=1}^{2}\left(\sum_{q} V_{r q}^{(2)}\left(x^{\prime}\right) \int_{-\infty}^{\infty} h_{t}^{*} \cdot\left\{i_{x} \times f_{r q}^{(2)}(y)\right\} d y\right. \\
+\sum_{s=0}^{2} \int_{\xi_{s}^{(2)}}^{\eta_{s}^{(2)}}\left[V_{r s}^{(2)}\left(x^{\prime}, \rho\right) \int_{-\infty}^{\infty} h_{t}^{*}\right. \\
\left.\left.\quad \cdot\left\{i_{x} \times f_{r s}^{(2)}(y, \rho)\right\} d y\right] d \rho\right) .
\end{aligned}
$$

The impedances to the $-x$ and $+x$ directions from the junction plane may be expressed as

$$
\begin{aligned}
V_{r q}^{(i)}\left(x^{\prime}\right) & =\mp Z_{r q \mp} I_{r q}^{(i)}\left(x^{\prime}\right) \\
V_{r s}^{(i)}\left(x^{\prime}, \rho\right) & =\mp Z_{r s \mp}(\rho) I_{r s}^{(i)}\left(x^{\prime}, \rho\right)
\end{aligned}
$$

where $Z_{r q \mp}$ and $Z_{r s \mp}(\rho)$ are the impedances of discrete modes and continuous spectrum to the $\mp x$ direction from the junction plane, respectively, and the upper sign (-) refers to $i=1$ and the lower sign ( + ) to $i=2$.

Defining the input impedance $Z_{\text {in, } q}\left(x^{\prime}\right)$ of the $\mathrm{TE}_{q}$ mode ( $q$ th TE mode) at $x=x^{\prime}-0$ to the right-hand side in Fig. 2 as

$$
Z_{\text {in }, q}\left(x^{\prime}\right)=V_{1 q}^{(1)}\left(x^{\prime}\right) / I_{1 q}^{(1)}\left(x^{\prime}\right)
$$

and using (10), (13), and (14), we obtain the following stationary expression:

$$
\begin{aligned}
Z_{\text {in }, q}\left(x^{\prime}\right)= & N_{1 q}^{2} Z_{1 q+}+Z \\
N_{1 q}= & \left|\int_{-\infty}^{\infty}\left\{i_{x} \times f_{1 q}^{(2)}(y)\right\}^{*} \cdot h_{t}(y) d y\right| \\
& \int_{-\infty}^{\infty}\left\{i_{x} \times f_{1 q}^{(1)}(y)\right\}^{*} \cdot h_{t}(y) d y \mid \\
Z= & \left\{\sum_{i=1}^{2} \sum_{r=1}^{2} \iint_{-\infty}^{\infty} h_{t}^{*}(y)\right. \\
& \cdot\left[\sum_{q^{\prime}} Z_{r q^{\prime} \mp}\left\{i_{x} \times f_{r q^{\prime}}^{(i)}(y)\right\}\left\{i_{x} \times f_{r q^{\prime}}^{(i)}\left(y^{\prime}\right)\right\}^{*}\right. \\
& +\sum_{s=0}^{2} \int_{\xi_{s}^{(i)}}^{\eta_{r s}^{(i)}} Z_{r s}(\rho)\left\{i_{x} \times f_{r s}^{(i)}(y, \rho)\right\} \\
& \left.\left.\cdot\left\{i_{x} \times f_{r s}^{(i)}\left(y^{\prime}, \rho\right)\right\}^{*} d \rho\right] \cdot h_{t}\left(y^{\prime}\right) d y d y^{\prime}\right\} \mid \\
& \left|\int_{-\infty}^{\infty}\left\{i_{x} \times f_{1 q}^{(1)}(y)\right\}^{*} \cdot h_{t}(y) d y\right|^{2}
\end{aligned}
$$

If we multiply $\left\{i_{x} \times(11)\right\}$ by $h_{t}^{*}$ and integrate over the In (16b), $q^{\prime} \neq q$ for $r=1$. 


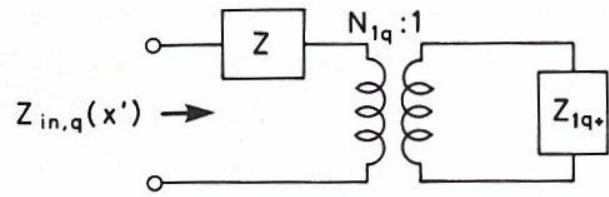

(a)

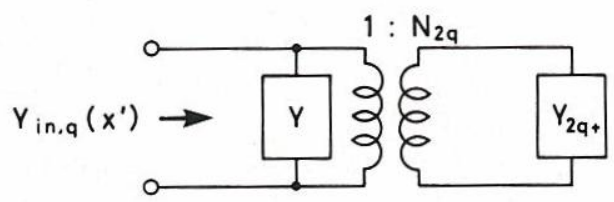

(b)

Fig. 3. Equivalent network for the step junction in Fig. 2. (a) $E_{p q}^{x}$ modes. (b) $E_{p q}^{y}$ modes.

Relation (15) can be interpreted in terms of a simple equivalent network as shown in Fig. 3(a). In the scalar wave approximation, the summation $\Sigma_{r=1}^{2}$ in $(16 \mathrm{~b})$ is removed and $r$ is replaced by 1 .

\section{B. $E_{p q}^{y}$ Modes}

Referring to (9)-(16), we obtain

$Y_{\text {in }, q}\left(x^{\prime}\right)=N_{2 q}^{2} Y_{2 q+}+Y$

$$
\begin{aligned}
N_{2 q}= & \left|\int_{-\infty}^{\infty}\left\{g_{2 q}^{(2)}(y) \times i_{x}\right\}^{*} \cdot e_{t}(y) d y\right| \\
& \int_{-\infty}^{\infty}\left\{g_{2 q}^{(1)}(y) \times i_{x}\right\}^{*} \cdot e_{t}(y) d y \mid \\
Y= & \left\{\sum_{i=1}^{2} \sum_{r=1}^{2} \iint_{-\infty}^{\infty} e_{t}^{*}(y)\right. \\
& \cdot\left[\sum_{q^{\prime}} Y_{r q^{\prime} \mp}\left\{g_{r q^{\prime}}^{(i)}(y) \times i_{x}\right\}\left\{g_{r q^{\prime}}^{(i)}\left(y^{\prime}\right) \times i_{x}\right\}^{*}\right. \\
& +\sum_{s=0}^{2} \int_{\xi_{s}^{(i)}}^{\eta_{s}^{(i)}} Y_{r s \mp}(\rho)\left\{g_{r s}^{(i)}(y, \rho) \times i_{x}\right\} \\
& \left.\left.\cdot\left\{g_{r s}^{(i)}\left(y^{\prime}, \rho\right) \times i_{x}\right\}^{*} d \rho\right] \cdot e_{t}\left(y^{\prime}\right) d y d y^{\prime}\right\} \\
& \left|\int_{-\infty}^{\infty}\left\{g_{2 q}^{(1)}(y) \times i_{x}\right\}^{*} \cdot e_{t}(y) d y\right|^{2}
\end{aligned}
$$

where $e_{t}$ is the unknown electric field in the junction plane $x=x^{\prime}$ in Fig. 2, $Y_{\mathrm{in}, q}\left(x^{\prime}\right)$ is the input admittance of the $\mathrm{TM}_{q}$ mode ( $q$ th TM mode) at $x=x^{\prime}-0$ to the right-hand side in Fig. 2, and $Y_{r q \mp}$ and $Y_{r s \mp}(\rho)$ are the admittances of discrete modes and continuous spectrum to the $\mp x$ direction from the junction plane, respectively. In (18b), $q^{\prime}$ $\neq q$ for $r=2$.

Relation (17) can be interpreted in terms of a simple equivalent network, as shown in Fig. 3(b). In the scalar wave approximation, the summation $\Sigma_{r=1}^{2}$ in (18b) is removed and $r$ is replaced by 2 .

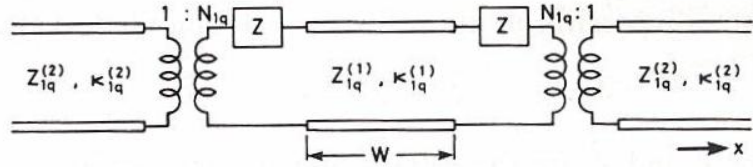

(a)

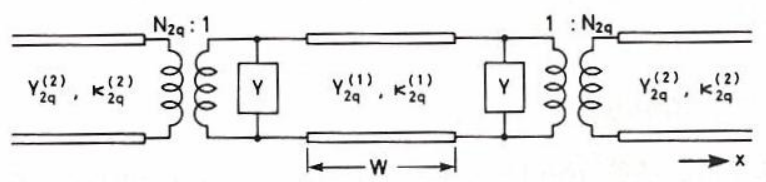

(b)

Fig. 4. Equivalent network for the dielectric waveguide in Fig. 1. (a) $E_{p q}^{x}$ modes. (b) $E_{p q}^{y}$ modes.

\section{Dispersion Relations}

Taking all of the contributions from the discrete and continuous parts of the spectrum and from the TE-TM coupling into account, we obtain the final equivalent networks as shown in Figs. 4(a) and (b) for the $E_{p q}^{x}$ and $E_{p q}^{y}$ modes in the original structure (Fig. 1), respectively. To evaluate the turns ratio $N_{r q}(r=1,2)$ of the transformer, the series impedance $Z$, and the shunt admittance $Y$ explicitly, we assume the form for the field $h_{t}$ or $e_{t}$ in the junction plane as

$$
\begin{array}{ll}
h_{t}=a_{1 q}^{(1)} g_{1 q}^{(1)}(y)+a_{1 q}^{(2)} g_{1 q}^{(2)}(y), & E_{p q}^{x} \text { modes } \\
e_{t}=a_{2 q}^{(1)} f_{2 q}^{(1)}(y)+a_{2 q}^{(2)} f_{2 q}^{(2)}(y), & E_{p q}^{y} \text { modes. }
\end{array}
$$

Using the Rayleigh-Ritz technique and (4), the ratio of variational parameters in (19), namely $a_{r q}^{(2)} / a_{r q}^{(1)} \equiv \bar{a}_{r q}^{(2)}$, is calculated by the following condition:

$$
\begin{array}{ll}
\partial Z_{\text {in }, q}\left(x^{\prime}\right) / \partial \bar{a}_{1 q}^{(2)}=0, & E_{p q}^{x} \text { modes } \\
\partial Y_{\text {in }, q}\left(x^{\prime}\right) / \partial \bar{a}_{2 q}^{(2)}=0, & E_{p q}^{y} \text { modes. }
\end{array}
$$

The value of $\bar{a}_{r q}^{(2)}$ allows the determination of the turns ratio, the series impedance, and the shunt admittance in Fig. 4.

For the waveguide having no discrete modes in region 2 , we assume the form for the field $h_{t}$ or $e_{t}$ to be $h_{t}=$ $g_{1 q}^{(1)}(y)$ or $e_{t}=f_{2 q}^{(1)}(y)$, respectively. In this case, $N_{1 q}=$ $N_{2 q}=0$, and $Z$ and $Y$ can be calculated directly from (16b) and (18b) without using (20), respectively.

\section{A. $E_{p q}^{x}$ Modes}

From the symmetry of the structure (Fig. 1), we recognize that two classes of solutions are possible: those ( $p$ $=1,3,5, \cdots)$ for which the main field components of the $E_{p q}^{x}$ modes, namely $E_{x}$ and $H_{y}$, are symmetric about the midplane $(x=0)$ and those $(p=2,4,6, \cdots)$ for which they are antisymmetric. The symmetric and antisymmetric modes then correspond to the free resonances of the short-circuit and open-circuit bisections of the equivalent network, respectively [14].

From the transverse resonance condition [9]-[18], we 
find the dispersion relation for the wavenumber $k_{z}$ to be

$$
\overleftarrow{Z}+\vec{Z}=0
$$

where $\overleftarrow{Z}$ and $\vec{Z}$ are the input impedances seen looking in opposite directions at $x=W / 2-0$ in the equivalent network.

\section{B. $E_{p q}^{y}$ Modes}

For the $E_{p q}^{y}$ modes, the symmetric and antisymmetric modes correspond to the free resonances of the open-circuit and short-circuit bisections of the equivalent network, respectively [14].

The dispersion relation is given by

$$
\stackrel{\leftarrow}{Y}+\stackrel{\vec{Y}}{=}=0
$$

where $\overleftarrow{Y}$ and $\vec{Y}$ are the input admittances seen looking in opposite directions at $x=W / 2-0$ in the equivalent network.

\section{Computed Results}

Fig. 5 shows dispersion characteristics for the $E_{p q}^{x}$ modes of the rib waveguide. Solid lines are the results of the vectorial wave analysis. Dashed lines are the results of the scalar wave analysis in [14]. Our results indicated by solid lines agree well with the results of the vectorial wave analysis using the mode-matching method [8]. The accuracy of the scalar wave analysis [14] becomes poorer for the higher-order modes.

Figs. 6(a) and (b) show dispersion characteristics for the $E_{p q}^{x}$ and $E_{p q}^{y}$ modes of the rectangular dielectric waveguide, respectively, where the normalized frequency $v$ and the normalized guide index $b$ are given by

$$
\begin{aligned}
& v=k_{0} t \sqrt{n_{1}^{2}-n_{2}^{2}} / \pi \\
& b=\left\{\left(k_{z} / k_{0}\right)^{2}-n_{2}^{2}\right\} /\left(n_{1}^{2}-n_{2}^{2}\right) .
\end{aligned}
$$

Solid lines are the results of the vectorial wave analysis. Dashed lines are the results of the scalar wave analysis in [17] and [18]. Effects of the TE-TM coupling are larger for the higher-order modes ( $E_{21}^{x}$ and $E_{21}^{y}$ modes) than for the fundamental modes ( $E_{11}^{x}$ and $E_{11}^{y}$ modes). Our results indicated by solid lines agree well with the results of the vectorial wave analysis using the collocation method [5].

Figs. 7 and 8 show dispersion characteristics of the embossed waveguide and of the embedded waveguide, respectively (vectorial wave analysis). Solid and dashed lines are for the $E_{p q}^{x}$ and $E_{p q}^{y}$ modes, respectively. Comparison of our results with the results of the vectorial wave analysis using the finite-element method [7] shows good agreement.

Table I shows the numerical results for the $E_{p q}^{x}$ modes of the optical strip waveguide, where $n_{1}^{(1)}=n_{1}^{(2)} \equiv n_{1}=$ $\sqrt{2.5}, n_{3}^{(1)}=n_{3}^{(2)}=n_{2}^{(1)} \equiv n_{2}=\sqrt{2.375}, n_{4}^{(1)}=n_{4}^{(2)}=$ $n_{2}^{(2)}=1.0, W=8 t_{1}$, and $t_{1}=t_{2} \equiv 2 t$. In Table I, the results of Marcatili's method [4], the effective index method [3], the variational method [6], and the finite-ele-

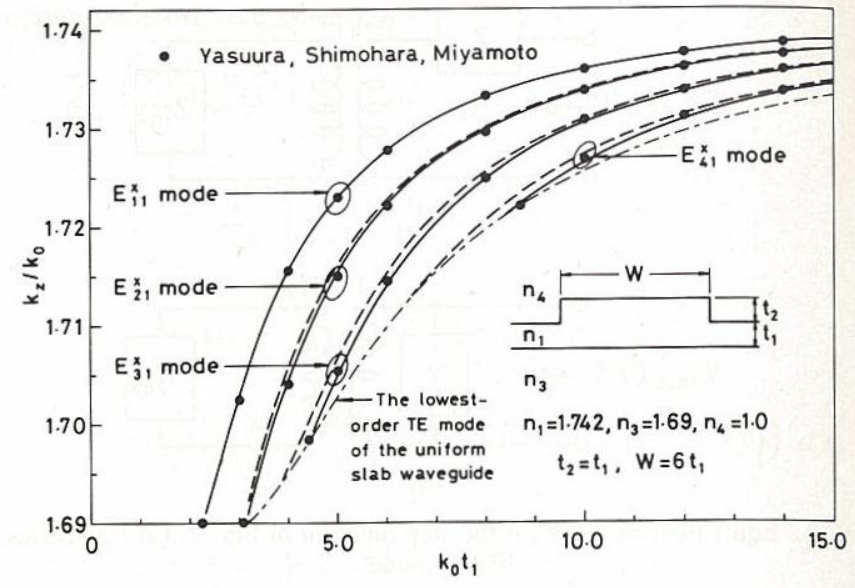

Fig. 5. Dispersion characteristics of the rib waveguide (vectorial wave analysis, solid lines; scalar wave analysis [14], dashed lines).

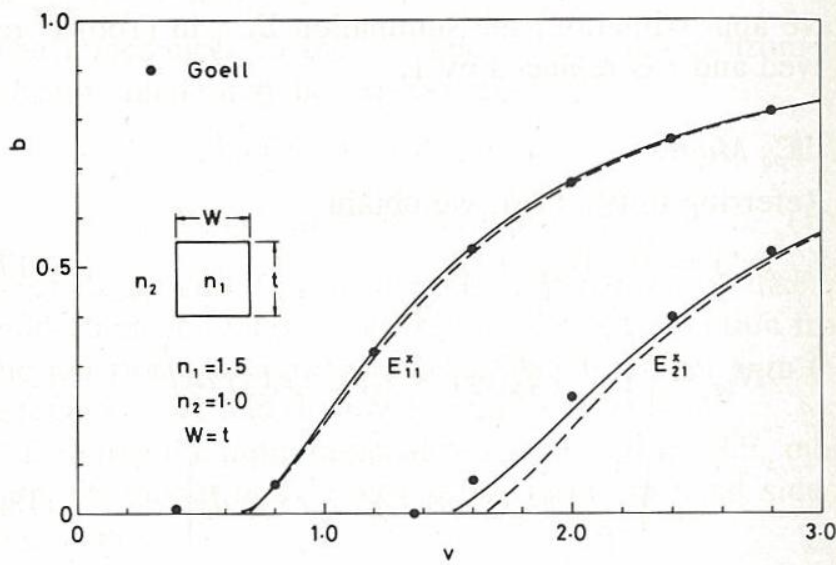

(a)

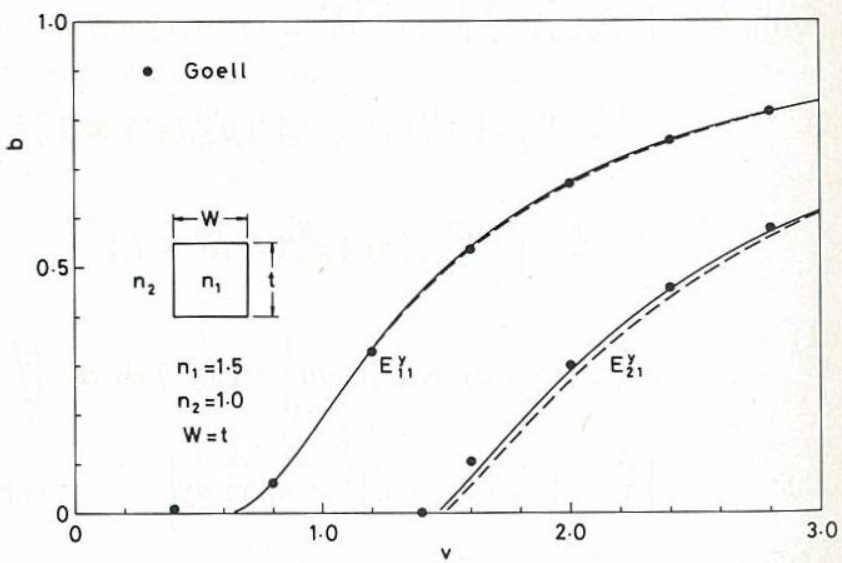

(b)

Fig. 6. Dispersion characteristics of the rectangular dielectric waveguide (vectorial wave analysis, solid lines; scalar wave analysis [17], [18]; dashed lines). (a) $E_{p q}^{x}$ modes. (b) $E_{p q}^{y}$ modes.

ment method [7] are also presented. Our results agree well with the results of the vectorial wave analyses using the variational method and the finite-element method. Our results obtained by neglecting the TE-TM coupling (scalar wave approximation) are given in the parentheses. Effects of the TE-TM coupling on the dispersion characteristics of this waveguide are very small, because the optical strip waveguide is formed by the dielectric strip that perturbs 


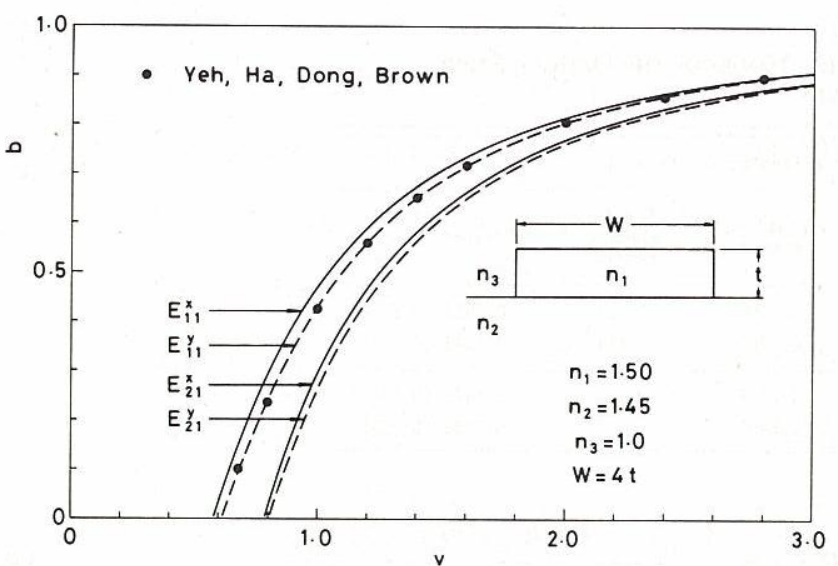

(a)

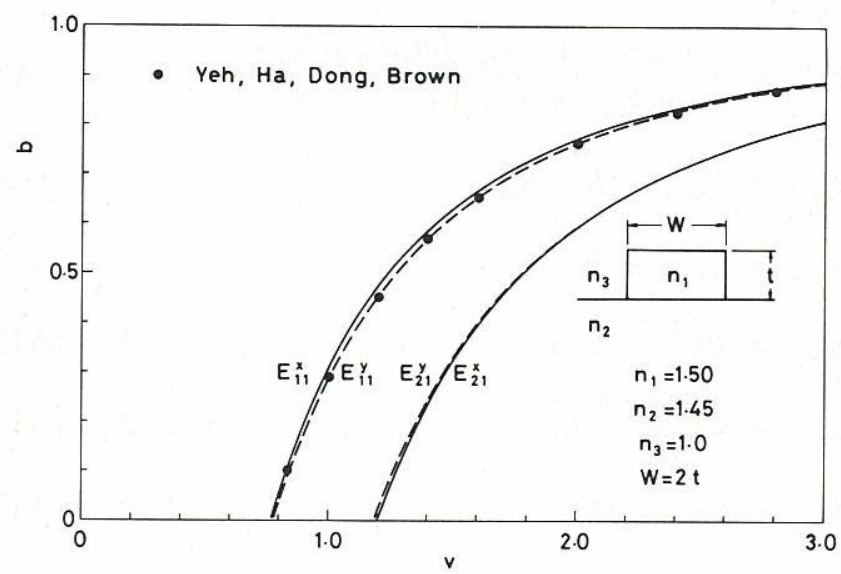

(b)

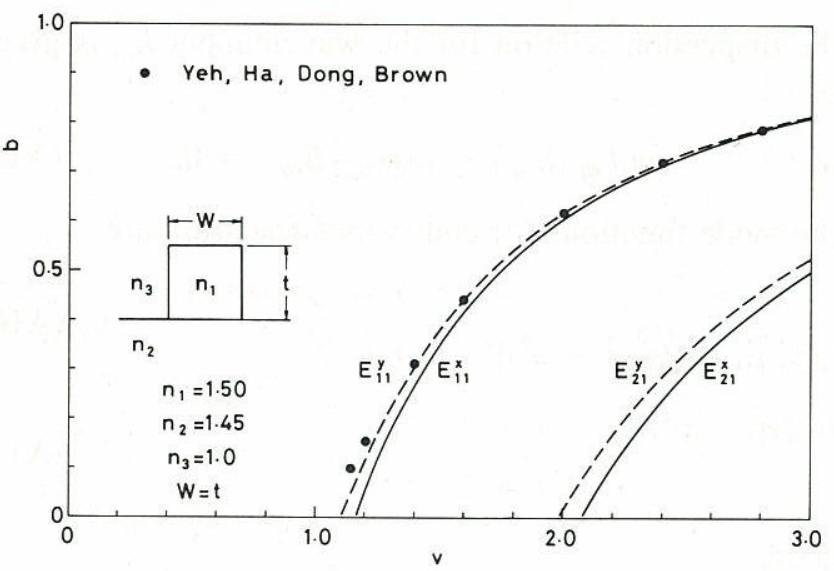

(c)

Fig. 7. Dispersion characteristics of the embossed waveguide $\left(E_{p q}^{x}\right.$ modes, solid lines; $E_{p q}^{y}$ modes, dashed lines). (a) $W=4 t$. (b) $W=2 t$. (c) $W=$ $t$.

the planar dielectric waveguide in which TE and TM modes are uncoupled.

\section{Conclusions}

Vectorial wave analysis of propagation characteristics of open dielectric waveguides for optical-integrated circuits has been performed by using an equivalent network approach. In this approach, all of the contributions from the discrete and continuous spectrum and from the TETM coupling, which are neglected in the earlier equivalent network approach, are taken into account, and there-

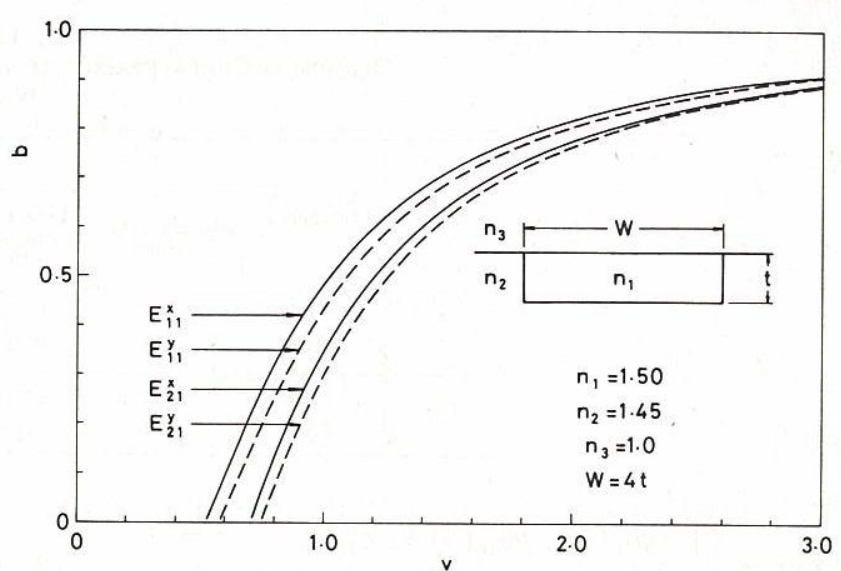

(a)

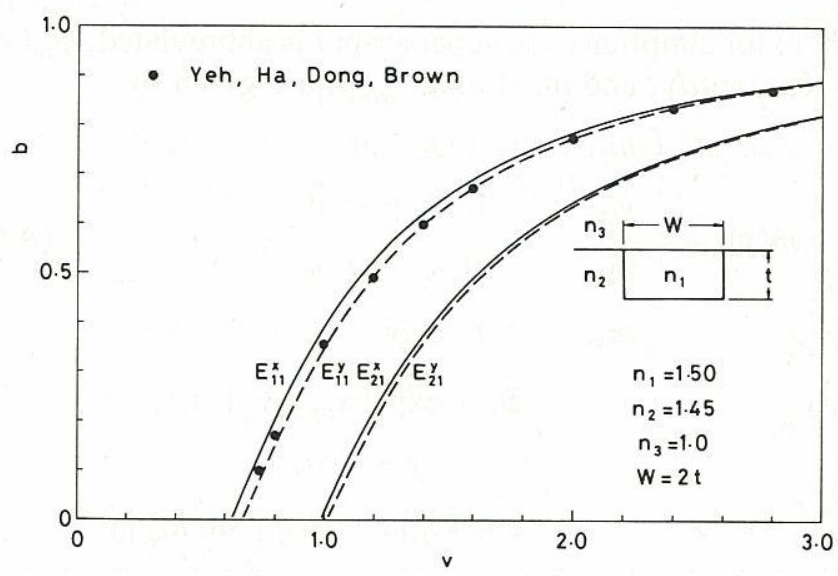

(b)

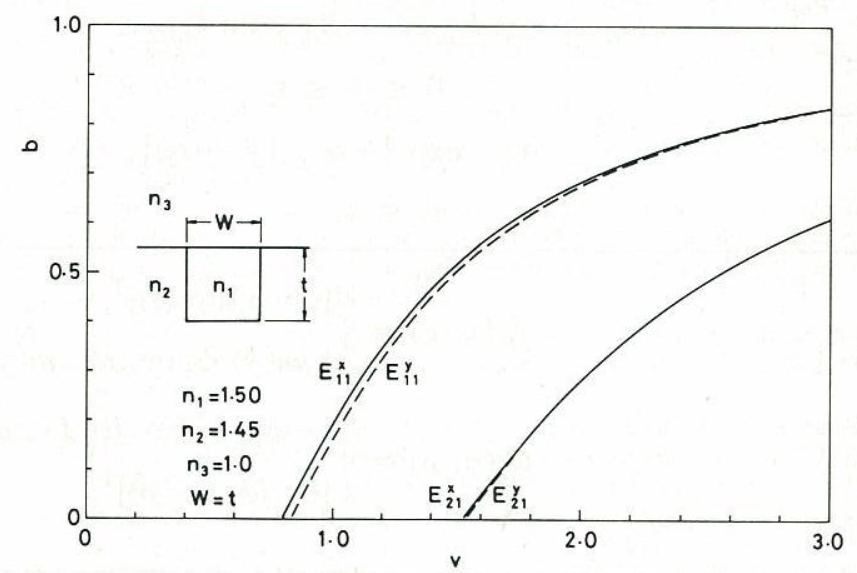

(c)

Fig. 8. Dispersion characteristics of the embedded waveguide $\left(E_{p q}^{x}\right.$ modes, solid lines; $E_{p q}^{y}$ modes, dashed lines). (a) $W=4 t$. (b) $W=2 t$. (c) $W=$ $t$.

fore the present equivalent network approach can give more accurate results for the various dielectric waveguides over a wide range of frequencies.

\section{APPENDIX}

The mode functions for discrete modes are

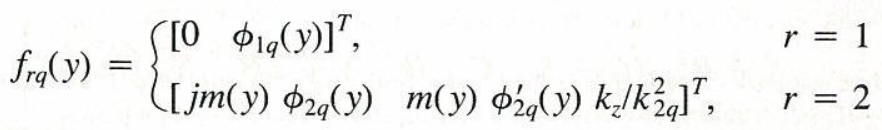


TABLE I

Dispersion Characteristics for the $E_{p q}^{x}$ MOdes of the Optical StRiP WAVEGUIDE

\begin{tabular}{|c|c|c|c|c|c|c|}
\hline \multirow[b]{2}{*}{ Mode } & \multirow{2}{*}{$\begin{array}{c}\text { Normalized } \\
\text { frequency } \\
v\end{array}$} & \multicolumn{5}{|c|}{ Normalized guide index b } \\
\hline & & $\begin{array}{l}\text { Marcatili 's } \\
\text { method }\end{array}$ & $\begin{array}{l}\text { Effective } \\
\text { index } \\
\text { method }\end{array}$ & $\begin{array}{l}\text { Variational } \\
\text { method }\end{array}$ & $\begin{array}{l}\text { Finite } \\
\text { element } \\
\text { method }\end{array}$ & Present analysis \\
\hline \multirow{2}{*}{$E_{11}^{x}$} & 0.25 & 0.167 & 0.278 & 0.2590 & 0.270 & $0.2676(0.2674)$ \\
\hline & 0.63 & 0.716 & 0.725 & 0.7226 & 0.724 & $0.7242(0.7242)$ \\
\hline \multirow{2}{*}{$E_{21}^{x}$} & 0.25 & 0.042 & 0.178 & 0.1313 & & $0.1476(0.1470)$ \\
\hline & 0.63 & 0.695 & 0.706 & 0.6995 & & $0.7036(0.7035)$ \\
\hline
\end{tabular}

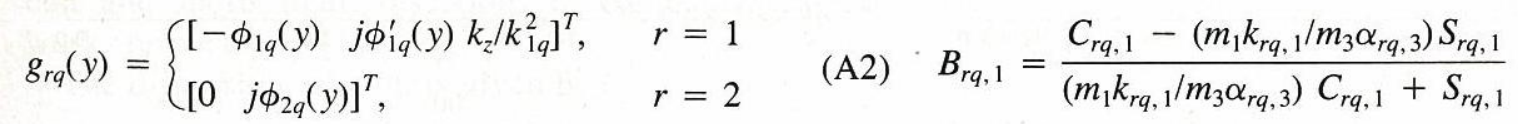

where for simplicity the superscript $i$ is abbreviated, $\phi_{r q}^{\prime}(y)$ $=d \phi_{r q}(y) / d y$, and $m(y)$ and $\phi_{r q}(y)$ are given by

$$
\begin{gathered}
m(y)=\left\{\begin{array}{cc}
m_{3}, & y \leq-t_{1} \\
m_{1}, & -t_{1} \leq y \leq 0 \\
m_{2}, & 0 \leq y \leq t_{2} \\
m_{4}, & t_{2} \leq y
\end{array}\right. \\
\phi_{r q}(y)=\frac{1}{\sqrt{D_{r q}}}\left\{\begin{array}{c}
B_{r q, 3} \exp \left\{\alpha_{r q, 3}\left(y+t_{1}\right)\right\}, \\
y \leq-t_{1} \\
\cos k_{r q, 1} y+B_{r q, 1} \sin k_{r q, 1} y, \\
-t_{1} \leq y \leq 0 \\
\cos k_{r q, 2} y+B_{r q, 2} \sin k_{r q, 2} y, \\
0 \leq y \leq t_{2} \\
A_{r q, 4} \exp \left\{-\alpha_{r q, 4}\left(y-t_{2}\right)\right\}, \\
t_{2} \leq y .
\end{array}\right.
\end{gathered}
$$$$
B_{r q, 2}=-\frac{C_{r q, 2}-\left(m_{2} k_{r q, 2} / m_{4} \alpha_{r q, 4}\right) S_{r q, 2}}{\left(m_{2} k_{r q, 2} / m_{4} \alpha_{r q, 4}\right) C_{r q, 2}+S_{r q, 2}}
$$

$C_{r q, j}=\cos k_{r q, j} t_{j}$,

$$
j=1,2 .
$$

The dispersion relation for the wavenumber $k_{r q}$ is given by

$$
m_{1} k_{r q, 1} B_{r q, 1}-m_{2} k_{r q, 2} B_{r q, 2}=0 .
$$

The mode functions for continuous spectrum are

$$
\begin{aligned}
& f_{r s}(y, \rho)= \begin{cases}{\left[\begin{array}{ll}
0 & \phi_{1 s}(y, \rho)
\end{array}\right]^{T},} & r=1 \\
{\left[\begin{array}{lll}
j m(y) \phi_{2 s}(y, \rho) & m(y) \phi_{2 s}^{\prime}(y, \rho) k_{z} /\left(k_{0}^{2} n_{3}^{2}-\rho^{2}\right)
\end{array}\right]^{T},} & r=2\end{cases} \\
& g_{r s}(y, \rho)= \begin{cases}{\left[\begin{array}{ll}
-\phi_{1 s}(y, \rho) & j \phi_{1 s}^{\prime}(y, \rho) k_{z} /\left(k_{0}^{2} n_{3}^{2}-\rho^{2}\right)
\end{array}\right]^{T},} & r=1 \\
{\left[\begin{array}{ll}
0 & j \phi_{2 s}(y, \rho)
\end{array}\right]^{T},} & r=2\end{cases}
\end{aligned}
$$

where

Here

$$
\begin{aligned}
& m_{j}= \begin{cases}1, & j=1,2,3,4, \\
1 / n_{j}^{2}, & r=1\end{cases} \\
& D_{r q}=\left(\frac{1}{2}\right)\left[m_{3} B_{r q, 3}^{2} / \alpha_{r q, 3}+m_{4} A_{r q, 4}^{2} / \alpha_{r q, 4}\right. \\
& +\sum_{j=1}^{2} m_{j}\left\{t_{j}+S_{r q, j} C_{r q, j} / k_{r q, j}\right. \\
& \left.\left.+B_{r q, j}^{2}\left(t_{j}-S_{r q, j} C_{r q, j} / k_{r q, j}\right)+2 B_{r q, j} S_{r q, j}^{2} / k_{r q, j}\right\}\right]
\end{aligned}
$$

$$
\phi_{r s}^{\prime}(y, \rho)=d \phi_{r s}(y, \rho) / d y .
$$

When $s=0\left(0 \leq \rho \leq k_{0} \sqrt{n_{3}^{2}-n_{4}^{2}}\right), \phi_{r s}(y, \rho)$ is given by

$\phi_{r s}(y, \rho)=\frac{1}{\sqrt{D_{r s}}}$

$$
\left\{\begin{array}{lc}
A_{r s, 3} \cos \rho\left(y+t_{1}\right)+B_{r s, 3} \sin \rho\left(y+t_{1}\right), & y \leq-t_{1} \\
\cos \sigma_{1} y+B_{r s, 1} \sin \sigma_{1} y, & -t_{1} \leq y \leq 0 \\
\cos \sigma_{2} y+B_{r s, 2} \sin \sigma_{2} y, & 0 \leq y \leq t_{2} \\
A_{r s, 4} \exp \left\{-\bar{\Delta}\left(y-t_{2}\right)\right\}, & t_{2} \leq y
\end{array}\right.
$$


Here

$$
\begin{gathered}
D_{r s}=m_{3}(\pi / 2)\left(A_{r s, 3}^{2}+B_{r s, 3}^{2}\right) \\
B_{r s, 1}=\left(m_{2} \sigma_{2} / m_{1} \sigma_{1}\right) B_{r s, 2} \\
B_{r s, 2}=-\left\{C_{2}-\left(m_{2} \sigma_{2} / m_{4} \bar{\Delta}\right) S_{2}\right\} / \\
, \quad\left\{\left(m_{2} \sigma_{2} / m_{4} \bar{\Delta}\right) C_{2}+S_{2}\right\} \\
B_{r s, 3}=\left(m_{1} \sigma_{1} / m_{3} \rho\right)\left(S_{1}+B_{r s, 1} C_{1}\right) \\
A_{r s, 3}=C_{1}-B_{r s, 1} S_{1} \\
A_{r s, 4}=C_{2}+B_{r s, 2} S_{2} \\
\bar{\Delta}=\sqrt{k_{0}^{2}\left(n_{3}^{2}-n_{4}^{2}\right)-\rho^{2}} \\
\sigma_{j}=\sqrt{k_{0}^{2}\left(n_{j}^{2}-n_{3}^{2}\right)+\rho^{2},} \quad j=1,2 \\
S_{j}=\sin \sigma_{j} t_{j}, \quad j=1,2 \\
C_{j}=\cos \sigma_{j} t_{j}, \quad j=1,2 .
\end{gathered}
$$

When $s=1$ and $2\left(k_{0} \sqrt{n_{3}^{2}-n_{4}^{2}} \leq \rho\right), \phi_{r s}(y, \rho)$ is given by

$$
\phi_{r s}(y, \rho)=\frac{1}{\sqrt{D_{r s}}}\left\{\begin{array}{lr}
A_{r s, 3} \cos \rho\left(y+t_{1}\right)+B_{r s, 3} \sin \rho\left(y+t_{1}\right), & y \leq-t_{1} \\
\cos \sigma_{1} y+B_{r s, 1} \sin \sigma_{1} y, & -t_{1} \leq y \leq 0 \\
\cos \sigma_{2} y+B_{r s, 2} \sin \sigma_{2} y, & 0 \leq y \leq t_{2} \\
A_{r s, 4} \cos \Delta\left(y-t_{2}\right)+B_{r s, 4} \sin \Delta\left(y-t_{2}\right), & t_{2} \leq y .
\end{array}\right.
$$

Here

$$
\begin{aligned}
D_{r s}= & (\pi / 2)\left\{m_{3}\left(A_{r s, 3}^{2}+B_{r s, 3}^{2}\right)\right. \\
& \left.+m_{4}(\Delta / \rho)\left(A_{r s, 4}^{2}+B_{r s, 4}^{2}\right)\right\} \\
B_{r s, 4}= & \left(m_{2} \sigma_{2} / m_{4} \Delta\right)\left(-S_{2}+B_{r s, 2} C_{2}\right) \\
\Delta= & \sqrt{k_{0}^{2}\left(n_{4}^{2}-n_{3}^{2}\right)+\rho^{2}}
\end{aligned}
$$

and $B_{r s, 3}, A_{r s, 3}$, and $A_{r s, 4}$ are the same as (A22), (A23), and (A24), respectively. $B_{r s, 1}$ and $B_{r s, 2}$ are given by

$$
\begin{aligned}
B_{r s, 1}= & \begin{cases}X+\sqrt{X^{2}+1}, & s=1 \\
X-\sqrt{X^{2}+1}, & s=2\end{cases} \\
B_{r s, 2}= & \left(m_{1} \sigma_{1} / m_{2} \sigma_{2}\right) B_{r s, 1} \\
X= & X_{N} / X_{D} \\
X_{N}= & m_{3}\left[\left\{\left(m_{1} \sigma_{1} / m_{3} \rho\right)^{2}-1\right\}\left(C_{1}^{2}-S_{1}^{2}\right)\right] \\
& +m_{4}(\Delta / \rho)\left[\left(m_{2} \sigma_{2} / m_{4} \Delta\right)^{2}\left\{\left(m_{1} \sigma_{1} / m_{2} \sigma_{2}\right)^{2} C_{2}^{2}-S_{2}^{2}\right\}\right. \\
& \left.-C_{2}^{2}+\left(m_{1} \sigma_{1} / m_{2} \sigma_{2}\right)^{2} S_{2}^{2}\right] \\
X_{D}= & 2\left[m_{3}\left\{\left(m_{1} \sigma_{1} / m_{3} \rho\right)^{2}-1\right\} S_{1} C_{1}\right. \\
& \left.+m_{4}(\Delta / \rho)\left(m_{1} \sigma_{1} / m_{2} \sigma_{2}\right)\left\{1-\left(m_{2} \sigma_{2} / m_{4} \Delta\right)^{2}\right\} S_{2} C_{2}\right]
\end{aligned}
$$

for $n_{1} \geq n_{2}$, and

$$
B_{r s, 1}=\left(m_{2} \sigma_{2} / m_{1} \sigma_{1}\right) B_{r s, 2}
$$

$$
\begin{aligned}
B_{r s, 2}= & \begin{cases}X+\sqrt{X^{2}+1}, & s=1 \\
X-\sqrt{X^{2}+1}, & s=2\end{cases} \\
X= & X_{N} / X_{D} \\
X_{N}= & m_{3}\left[\left(m_{1} \sigma_{1} / m_{3} \rho\right)^{2}\left\{\left(m_{2} \sigma_{2} / m_{1} \sigma_{1}\right)^{2} C_{1}^{2}-S_{1}^{2}\right\}\right. \\
& \left.-C_{1}^{2}+\left(m_{2} \sigma_{2} / m_{1} \sigma_{1}\right)^{2} S_{1}^{2}\right] \\
& +m_{4}(\Delta / \rho)\left[\left\{\left(m_{2} \sigma_{2} / m_{4} \Delta\right)^{2}-1\right\}\left(C_{2}^{2}-S_{2}^{2}\right)\right] \\
X_{D}= & 2\left[m_{3}\left(m_{2} \sigma_{2} / m_{1} \sigma_{1}\right)\left\{\left(m_{1} \sigma_{1} / m_{3} \rho\right)^{2}-1\right\} S_{1} C_{1}\right. \\
& \left.+m_{4}(\Delta / \rho)\left\{1-\left(m_{2} \sigma_{2} / m_{4} \Delta\right)^{2}\right\} S_{2} C_{2}\right]
\end{aligned}
$$

for $n_{1} \leq n_{2}$.

\section{ACKNOWLEDGMENT}

The authors wish to thank H. Ishii and T. Hayashi for their assistance in numerical computations. 
New physical effects,' IEEE Trans. Microwave Theory Tech., vol. MTT-29, pp. 855-869, Sept. 1981.

[14] M. Koshiba and M. Suzuki, "Equivalent network analysis of dielectric thin-film waveguides for optical-integrated circuits and its applications,"' Radio Sci., vol. 17, pp. 99-107, Jan.-Feb. 1982.

[15] N. Dagli and C. G. Fonstad, "Analysis of rib waveguides with sloped rib sides," Appl. Phys. Lett., vol. 46, pp. 529-531, Mar. 1985.

[16] N. Dagli and C. G. Fonstad, "Analysis of rib dielectric waveguides,"' IEEE J. Quantum Electron., vol. QE-21, pp. 315-321, Apr. 1985.

[17] M. Koshiba, H. Ishii, and M. Suzuki, "Improved equivalent network analysis of a dielectric waveguide placed on a ground plane," Trans. Inst. Electron. Commun. Eng. Japan, vol. E65, pp. 572-578, Oct. 1982:

[18] M. Koshiba, H. Ishii, and M. Suzuki, "Simple equivalent network for a rectangular dielectric image guide," Electron. Lett., vol. 18, pp. 473-474, May 1982.

[19] D. Marcuse, Theory of Dielectric Optical Waveguides. New York: Academic, 1974.

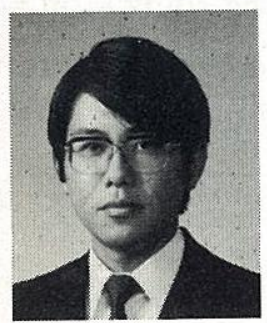

Masanori Koshiba (SM'84) was born in Sapporo, Japan, on November 23, 1948. He received the B.S., M.S., and Ph.D. degrees in electronic engineering from Hokkaido University, Sapporo, Japan, in 1971, 1973, and 1976, respectively.

In 1976, he joined the Department of Electronic Engineering, Kitami Institute of Technology, Kitami, Japan. Since 1979, he has been an Assistant Professor of Electronic Engineering at Hokkaido University. He has been engaged in research on surface acoustic waves, dielectric opti- cal waveguides, and applications of finite-element and boundary-element methods to field problems.

Dr. Koshiba is a member of the Institute of Electronics and Communication Engineers of Japan, the Institute of Television Engineers of Japan, the Institute of Electrical Engineers of Japan, the Japan Society for Simulation Technology, and the Japan Society for Computational Methods in Engineering.

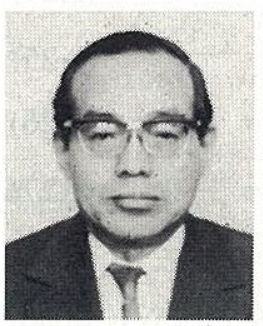

Michio Suzuki (SM'57) was born in Sapporo, Japan, on November 14, 1923. He received the B.S. and Ph.D. degrees in electrical engineering from Hokkaido University, Sapporo, Japan, in 1946 and 1960 , respectively.

From 1948 to 1962, he was an Assistant Professor of Electrical Engineering at Hokkaido University. Since 1962, he has been a Professor of Electronic Engineering at Hokkaido University. From 1956 to 1957, he was a Research Associate at the Microwave Research Institute of Polytechnic Institute of Brooklyn, Brooklyn, NY.

Dr. Suzuki is a member of the Institute of Electronics and Communication Engineers of Japan, the Institute of Electrical Engineers of Japan, the Institute of Television Engineers of Japan, the Japan Society of Information and Communication Research, and the Japan Society for Simulation Technology. 